\title{
Genetic analysis of the role of $A / x 4$ in the coordination of lower body and external genitalia formation
}

\author{
Daisuke Matsumaru ${ }^{1,2,8}$, Ryuma Haraguchi ${ }^{1,2,3,8}$, Anne M Moon ${ }^{4}$, Yoshihiko Satoh ${ }^{1,2}$, Naomi Nakagata ${ }^{5}$, \\ Ken-ichi Yamamura ${ }^{6}$, Naoki Takahashi ${ }^{7}$, Sohei Kitazawa ${ }^{3}$ and Gen Yamada ${ }^{\star, 1,2}$
}

\begin{abstract}
Although several syndromes include abnormalities of both the ventral body wall and external genitalia, the developmental bases of this correlation are largely unknown. Naturally occurring mutations in Aristaless-like 4 (Alx4, Strong's luxoid: Alx4 $4^{L s t}$ ) have ventral body wall and pelvic girdle abnormalities. We sought to determine whether the development of the genital tubercle (GT) and its derivatives, the external genitalia, is affected by this mutation. We thus performed genetic and tissue labeling analyses


cell migration during GT formation by tissue labeling experiments and discovered that the cells located in the proximal segment of the umbilical cord (infra-umbilical mesenchyme) migrate toward the dorsal part of the GT. The Alx4Lst/Lst mutants also displayed augmented expression of $\mathrm{Hh}$ signal-related genes. Hence, we analyzed a series of combinatorial mutants for $A / x 4$, Sonic hedgehog (Shh) and GLI-Kruppel family member 3 (Gli3). These phenotype-genotype analyses suggested a genetic interaction between $A / x 4$ and Hh signaling during GT formation. Moreover, Hh gain-of-function mutants phenocopied some of these phenotypes. These observations reveal novel information regarding the pathogenic mechanisms of syndromic lower ventral body malformations, which are largely unknown.
\end{abstract}

European Journal of Human Genetics (2014) 22, 350-357; doi:10.1038/ejhg.2013.160; published online 14 August 2013

Keywords: Alx4; Hedgehog signaling; external genitalia; cell migration; genetic analysis

\section{INTRODUCTION}

Organogenesis is a developmentally coordinated process such that the adjacent tissues and structures influence one another's morphogenesis. For instance, bladder exstrophy (OMIM: \%600057) is associated not only with defects in the bladder wall but also in the external genitalia and the pelvic girdle. ${ }^{1-4}$ The ventral body wall defect called omphalocele is also associated with cardiac and anorectal malformations in more than $50 \%$ of cases. ${ }^{5,6}$ Although methodologies for genetic analysis have been improved recently, the pathogenic mechanisms of such syndromic malformations are unclear. ${ }^{7-9}$

The genital tubercle (GT) is the anlage of the external genitalia in both males and females. The GT is composed of a urethral plate/tube derived from the cloacal membrane together with the mesenchyme and the outer ectoderm. ${ }^{10}$ Congenital abnormalities frequently result from abnormal GT formation and its subsequent development. A common defect is hypospadias, in which the urethra opens aberrantly into the ventral GT. ${ }^{11}$ Recent studies have identified the causative genes and signaling pathways related to hypospadias-like pathogenesis, including Hedgehog $(\mathrm{Hh})$ and Wnt. ${ }^{12-19}$ On the other hand, epispadias is a congenital abnormality reflecting aberrant development of the dorsal part of the GT. Patients with epispadias often present with other abnormalities such as bladder exstrophy. ${ }^{2,3}$ The incidence of dorsal GT abnormalities including epispadias
(2.4 in 100000) is rare relative to hypospadias (1 per 300 live births). ${ }^{2,4}$ Hence, the pathogenic mechanisms underlying dorsal GT hypoplasia are poorly understood and require investigation.

The Aristaless-like homeobox 4 (Alx4) is an essential gene for skull and appendage development in humans and mouse. ${ }^{20-23}$ Several heterozygous and homozygous mutations in the human ALX4 gene have been identified and the human phenotypes vary with the type of mutations. ${ }^{22-24}$ Common defects observed with ALX4 mutations in both heterozygotes and homozygotes are parietal foramina, which are oval defects of the parietal bones. Affected individuals also display alopecia, coronal craniosynostosis, hypertelorism, severely depressed nasal bridge and ridge, bifid nasal tip, hypogonadism/cryptorchidism, callosal body agenesis and/or mental retardation. ${ }^{25}$ Several mouse Alx4 mutants have also been reported. ${ }^{26}$ Representative phenotypes include preaxial polydactyly, hemimelia of the tibia, craniofacial abnormalities (reduction of the parietal and frontal bones), eyelid abnormalities, ventral body wall defects, cryptorchidism and alopecia. $^{20,21,26}$

One of the Alx4 mouse mutants, Alx $4^{\text {Lst }}$ (Strong's luxoid: Lst ${ }^{\top}$ ), is a naturally occurring 16-bp deletion in the homeobox-encoding domain. ${ }^{26,27}$ Such deletion disrupts the DNA recognition helix and results in a frame-shift of the downstream protein-coding sequence. ${ }^{26}$ We previously utilized combinatorial mutants of $A l x 4^{L s t}, G l i 3^{X t}$ and

${ }^{1}$ Department of Developmental Genetics, Institute of Advanced Medicine, Wakayama Medical University, Wakayama, Japan; ${ }^{2}$ Institute of Molecular Embryology and Genetics, Kumamoto University, Kumamoto, Japan; ${ }^{3}$ Department of Molecular Pathology, Ehime University Graduate School of Medicine, Ehime, Japan; ${ }^{4}$ Weis Center for Research, Geisinger Clinic, Danville, PA, USA; ${ }^{5}$ Division of Reproductive Engineering, Center for Animal Resources and Development, Kumamoto University, Kumamoto, Japan; ${ }^{6}$ Division of Developmental Genetics, Institute of Resource Development and Analysis, Kumamoto University, Kumamoto, Japan; 7 Department of Applied Biological Chemistry, Graduate School of Agricultural and Life Sciences, The University of Tokyo, Tokyo, Japan

${ }^{8}$ These authors contributed equally to this work.

*Correspondence: Professor G Yamada, Department of Developmental Genetics, Institute of Advanced Medicine, Wakayama Medical University, 811-1 Kimiidera, Wakayama 641-8509, Japan. Tel: +81 73499 5026; Fax: +81 73499 5026; E-mail: transg8@wakayama-med.ac.jp or genyama77@yahoo.co.jp

Received 10 October 2012; revised 11 April 2013; accepted 8 May 2013; published online 14 August 2013 
Shh to show that omphalocele pathogenesis likely relates to disruption of the ventral body wall and pelvic girdle formation. ${ }^{28}$ Here we investigate the correlation of these phenotypes with the broad spectrum of lower body abnormalities, including GT morphogenesis. We analyzed the function of Alx4 during GT formation with a series of genetically modified mouse models and tissue labeling experiments. We found that the Alx4 ${ }^{\text {Lst }}$ compound mutants display dorsal GT hypoplasia and some of the above defects could be remedied by modulating Hh signaling. In addition, tissue labeling and extirpation experiments revealed that mesenchymal cells located in the proximal umbilical cord normally migrate to the dorsal GT. These results suggest an essential interaction between Alx4 function and $\mathrm{Hh}$ signaling for coordinated development of lower ventral body structures.

\section{MATERIALS AND METHODS}

Mice

The mutant mice used herein were Shh, Gli3 ${ }^{X t}\left(X t^{\top}\right)$, Alx $4^{L s t}\left(L s t^{\top}\right)$, CAGGSCreER ${ }^{T M}$ and Rosa26-SmoM2. ${ }^{26,27,29-32}$ The genotypes of each strain were determined as reported previously. To obtain Alx $4^{L s t}$; Gli3 ${ }^{X t}$; Shh combinatorial mutants, single, double or triple heterozygous male and female mice were crossed. Noon of the day when the vaginal plug was identified was considered as embryonic day 0.5 (E0.5). All experimental procedures and protocols were approved by the Committees on Animal Research at Wakayama Medical University and at Kumamoto University.

\section{Histological analyses}

Mouse embryos were fixed overnight in $4 \%$ paraformaldehyde (Sigma, St Louis, MO, USA) with phosphate-buffered saline, dehydrated through methanol, embedded in paraffin, and $8 \mu \mathrm{m}$ serial sections were prepared. Hematoxylin and eosin staining was performed using standard procedures. ${ }^{14,17}$ Immunohistochemical analysis was performed by standard procedures using anti-fibronectin antibody (1:500, Sigma). Signal amplification was performed using VECTASTAIN ABC Rabbit IgG Kit (Vector Laboratories, Burlingame, CA, USA) and the immunocomplexes were detected with DAB staining.

\section{Statistical analysis}

For the statistical analyses of the length between the umbilical cord and the GT, the length was measured with a slide gauge. Data were analyzed using Student's $t$-test (two tailed). $P<0.001$ was considered to be statistically significant. Values are given as means $\pm \mathrm{SD}$.

\section{In situ hybridization}

Whole-mount and section in situ hybridizations for gene expression analyses were performed as previously described. ${ }^{28}$ The antisense riboprobe templates have been described previously: Alx4 (kindly provided by Dr Toshihiko Shiroishi and Dr Frits Meijlink), transcription factor AP-2 alpha (Ap2 $\alpha)$ (kindly provided by Dr Chi-chung Hui), Mab-21 (male abnormal 21: C. elegans) like 2 (Mab21l2), ${ }^{33}$ paired-like homeodomain transcription factor 1 (Pitxl) (kindly provided by Dr YiPing Chen), fibroblast growth factor 8 ( Fgf8) (kindly provided by Dr Brigid LM Hogan), Sonic hedgehog (Shh) (kindly provided by Dr Chisa Shukunami) and Patched homolog 1 (Ptc1) (kindly provided by Dr Jun Motoyama).

\section{Hh signaling gain-of-function analysis}

Rosa26-SmoM2 (R26-SmoM2) homozygous female mice were crossed with male mice bearing the tamoxifen-inducible CAGGS-CreER ${ }^{T M}$ transgene. ${ }^{31,32,34}$ Pregnant R26-SmoM2 females were treated with tamoxifen ( $2 \mathrm{mg}$ per $40 \mathrm{~g}$ maternal body weight) orally using a gavage needle. No overt teratological effects of tamoxifen were observed in the control embryos under these conditions. ${ }^{13-15}$

\section{DiI tissue labeling}

The harvested embryos at E12.0 were embedded in saline with 3\% agarose and sectioned sagittally using a vibratome. The DiI solution $(0.01 \%$ DiI dissolved in dimethylsulfoxide) was injected into the proximal umbilical mesenchyme with a glass needle.

\section{Explanted tissue cultures}

DiI-labeled and dissected tissue samples were cultured for 36 or $48 \mathrm{~h}$ on ISOPORE membrane filters (Millipore, Bedford, MA, USA) in BGJb medium (Gibco BRL, Carlsbad, CA, USA) with $10 \mathrm{~mm}$ HEPES (pH 7.4) and $0.1 \mathrm{mg} / \mathrm{ml}$ L-ascorbic acid at $37^{\circ} \mathrm{C}$ in air and $5 \% \mathrm{CO}_{2}$.

\section{RESULTS}

Alx $4^{\text {Lst/Lst }}$ mutants have hypoplasia of the dorsal GT

To gain insight into the role of Alx4 during body wall and GT formation, we examined the expression of Alx4 in the body wall and urogenital organs including GT in E10.5-13.5 wild-type embryos. Alx4 expression was observed around the proximal umbilical cord region at E10.5, before GT protrusion (Figures 1a and $\mathrm{a}^{\prime}$ ). At E11.5, Alx4 expression was prominent at the dorsal GT mesenchyme and the ventral body wall region (Figures $1 b$ and $b^{\prime}$ ). Expression in the proximal umbilical mesenchyme persisted until E12.5 and then gradually decreased later (Figures $1 \mathrm{c}^{\prime}$ and $\mathrm{d}^{\prime}$ ). Expression in the dorsal GT mesenchyme was observed at E12.5 and E13.5 (Figures 1c- $\mathrm{d}^{\prime}$ ).

Urogenital phenotypes of $A l x 4^{\text {Lst/Lst }}$ mutants were apparent by E11.5 (Figures 1e and $\mathrm{j}$ ). The proximal umbilical mesenchyme and dorsal GT were significantly hypoplastic in Alx $4^{\text {Lst/Lst }}$ mutants at E11.5 and E12.5 (Figures $1 \mathrm{j}$ and $\mathrm{k}$; blue arrowheads). This hypoplastic region corresponds to an Alx4 expression domain (Figures $1 b^{\prime}$ and $c^{\prime}$ ). In the late fetal stages, Alx $4^{\text {Lst/Lst }}$ mutants displayed pelvic girdle abnormalities and ventral body wall defects such as omphalocele, thin body wall and bladder wall hypoplasia (Figure 11; blue arrow, data not shown). The dorsal GT was hypoplastic in the mutants at E18.5 (Figures 11-n; red arrowheads). Alx $4^{\text {Lst/Lst }}$ mutants also had reduced size of glans penis and dorsal preputial tissues (Figures 1l-n). These results indicate that Alx4 function is essential for the normal formation of the caudal regions of the embryo.

\section{Expression of several differentiation markers is altered in $A l x 4^{L s t / L s t}$ mutants}

Ap $2 \alpha$ function is known to be essential for the formation of the ventral body wall and GT. ${ }^{35-37}$ The Ap2 $\alpha$-positive domain in the dorsal GT mesenchyme was smaller in $A l x 4^{\text {Lst } / L s t}$ mutants compared with controls at E12.0 (Figures 2a and b; red arrowheads). Pitx1 gene is also expressed in the dorsal GT and proximal umbilical mesenchyme (Figure 2c). Such expression was retained at a high level in the dorsal GT and reduced in the proximal umbilical mesenchyme of Alx $4^{L s t / L s t}$ mutants (Figure 2d; red arrowheads). Expression of Mab21l2 gene and fibronectin protein (detected using immunohistochemistry) was markedly decreased throughout the GT in Alx $4^{\text {Lst } / L s t}$ mutants (Figures 2e-h; red arrowheads). Mab21l2 gene is expressed in the proximal umbilical mesenchyme and the dorsal GT at E12.5 (Figure 2e). Congenital body wall defects due to Mab21l2 mutation have been reported. ${ }^{33}$

Previous reports have suggested that $A l x 4$ gene function is related to Hh signaling, such as in the developing limb. ${ }^{20,27,28,38,39}$ Hence, we analyzed the expression of genes in the Hh pathway. The expression of $S h h$, one of the Hh ligands, was increased in the mutant cloacal epithelium (Figure 2j; red arrow). Ptcl encodes the receptor for $\mathrm{Hh}$ ligands, and is a downstream target of Hh signaling; ${ }^{40,41}$ its expression 

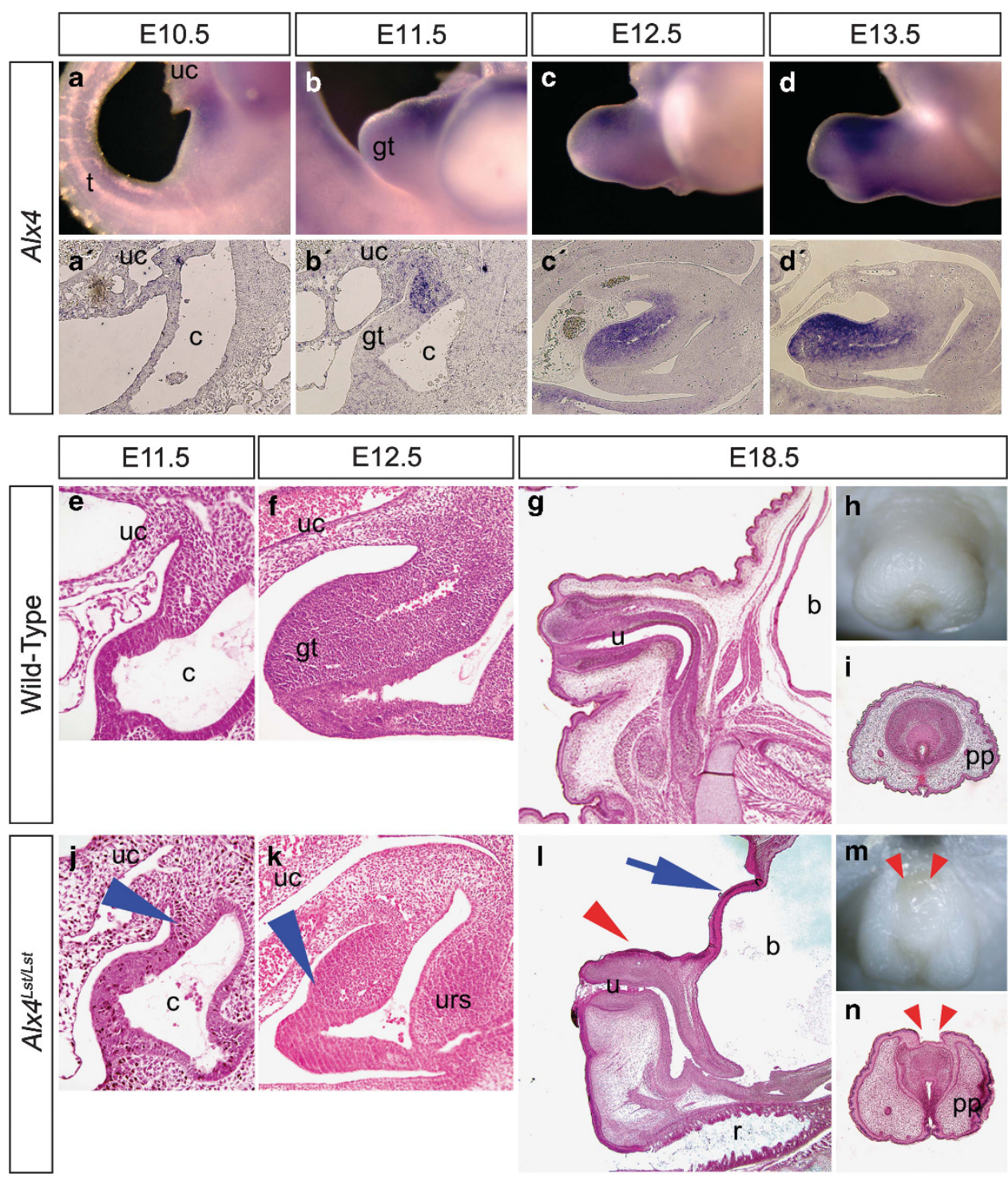

Figure 1 Expression of $A / x 4$ and urogenital abnormalities of $A / x 4^{L s t / L s t}$ mutants. Lateral view of $A / x 4$ gene expression in whole-mount embryos at E10.5-E13.5 (a-d). Alx4 expression in sagittal sections at E10.5-E13.5 (a'-d'). Sagittal sections of urogenital organs of wild-type embryos (e-g) and Alx4 ${ }^{\text {Lst/Lst }}$ mutants (j-I) at E11.5, E12.5 and E18.5. Blue arrowheads indicate dorsal hypoplasia of the GT in Alx4 $4^{\text {Lst/Lst }}$ mutants. Sagittal sections (g, I), frontal view $(\mathbf{h}, \mathbf{m})$ and coronal sections $(\mathbf{i}, \mathbf{n})$ of the GT in E18.5 embryos. Red arrowheads in I-n indicate hypoplastic dorsal GT in Alx4 ${ }^{L s t / L t}$ mutants at E18.5. Blue arrow in I indicates hypoplastic ventral body wall of $A / x 4^{L \text { st } / \text { Lst }}$ mutants. b: bladder, c: cloaca, gt: genital tubercle, pp: prepuce, $r$ : rectum, t: tail, u: urethra, uc: umbilical cord, urs: urorectal septum.

was also increased in the cloacal mesenchyme of the mutants (Figure 2l; red arrows).

Migration of proximal umbilical mesenchymal cells is impaired by Alx4 mutation

We observed a significant reduction in the distance between the umbilical cord and GT in Alx $4^{\text {Lst/Lst }}$ mutants at E18.5 (Figure 2m; wild-type versus $A l x 4^{\text {Lst } / \text { Lst }}$ mutants: $1.75 \pm 0.42, n=10$ versus $0.54 \pm 0.09, n=5 ; P<0.001)$. The distance between the fetal umbilical cord and the GT in early gestation has been suggested to be useful for early pre-natal diagnosis of congenital bladder exstrophy. ${ }^{42}$ As Fibronectin (Fnl) is an extracellular matrix component essential for cell adhesion and migration, ${ }^{43}$ and its expression was decreased in Alx $4^{L s t / L s t}$ mutants (Figure $2 \mathrm{~h}$; red arrowheads), the decreased distance between the umbilical cord and the GT prompted us to examine cell migration during the GT formation. We first performed tissue extirpation experiments to determine the significance of the cells in the proximal umbilical mesenchyme. The lower body tissues from E12.5 wild-type embryos with or without proximal umbilical mesenchyme were cultured in the BGJb medium (Figures $3 \mathrm{a}$ and c). After $48 \mathrm{~h}$ in culture, we examined the gross morphology of the control tissue versus that lacking umbilical cord (Figures $3 \mathrm{~b}$ and $\mathrm{d}$ ). We observed dorsal GT hypoplasia in explants cultured without proximal umbilical mesenchyme (Figure $3 \mathrm{~d}$; red arrow). We next performed tissue labeling experiments. Sagittally sliced tissues were labeled with DiI at E12.0 and incubated for $36 \mathrm{~h}$. We observed the DiI-labeled cells distributed from the proximal umbilical mesenchyme to the dorsal part of GT (Figures $3 \mathrm{e}-\mathrm{f}^{\prime}$ ). When we performed this 

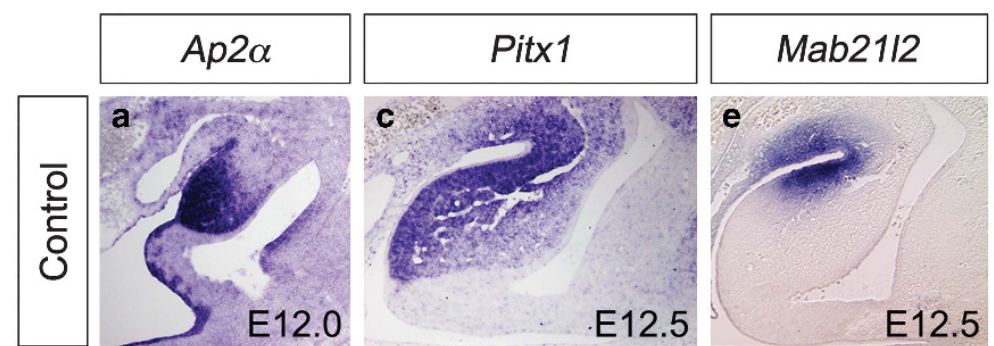

\section{Fibronectin}
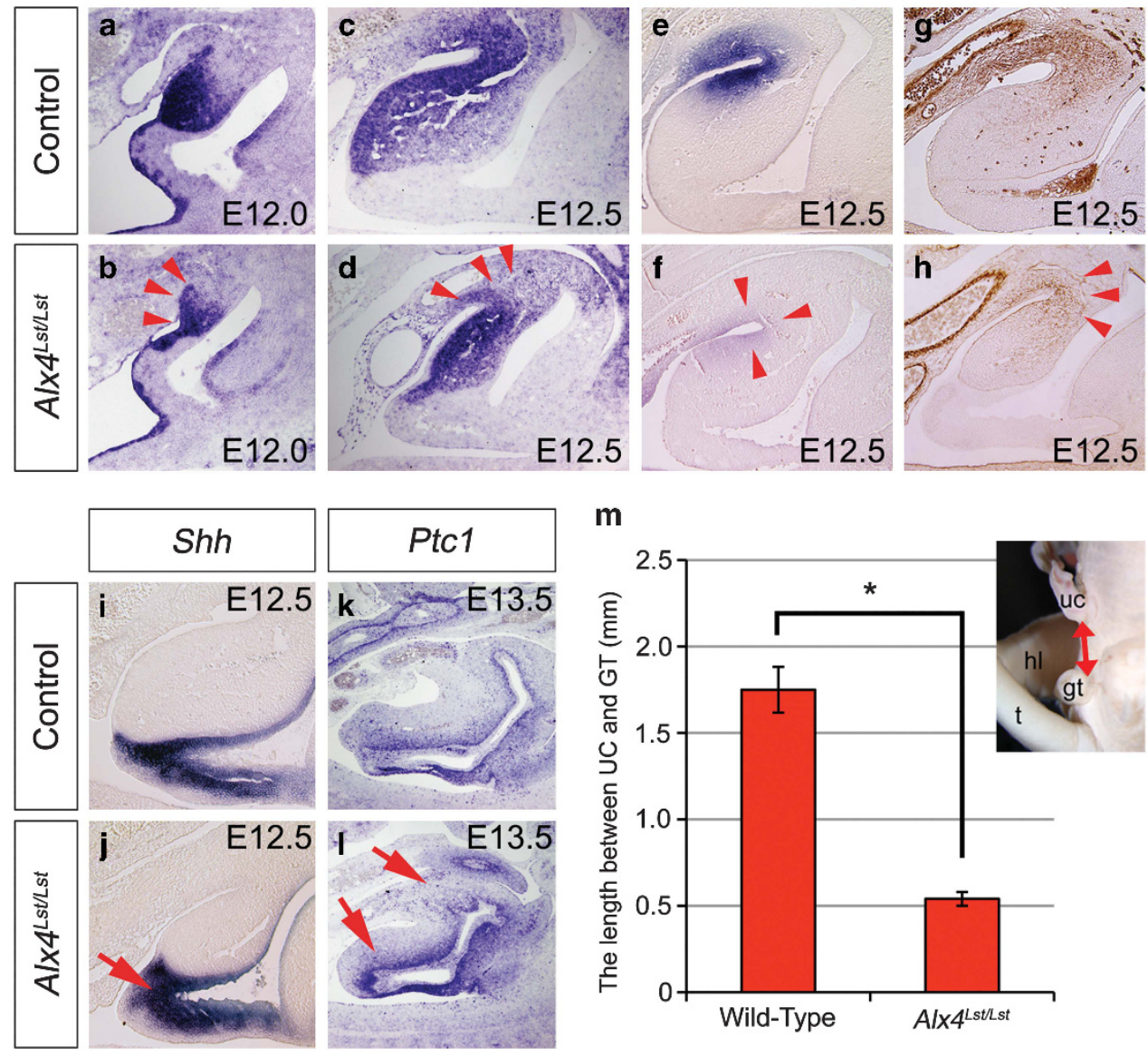

m

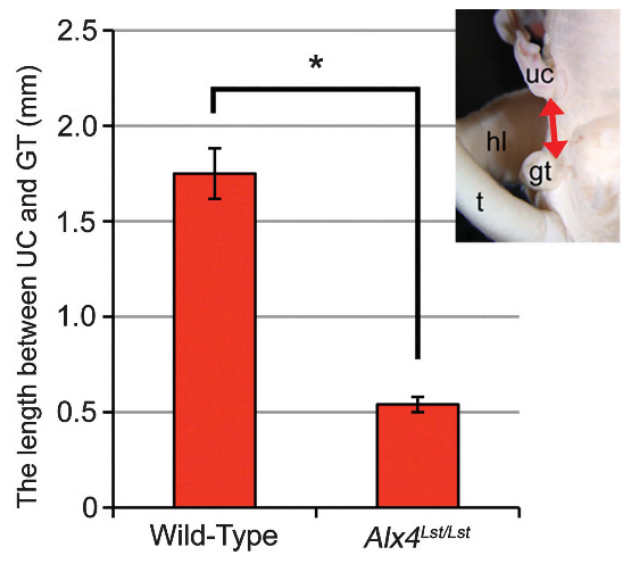

Figure 2 Expression of developmental markers in Alx $4^{L s t / L s t}$ mutants. Sagittal sections of the control and $A / x 4^{L s t / L s t}$ mutants (a-I). Expression of Ap2a (a, b), Pitx1 (c, d) and Mab21/2 (e, f) genes was examined at E12.0 or E12.5. Immunohistochemistry to detect Fibronectin protein was performed in E12.5 sagittal sections $(\mathbf{g}, \mathbf{h})$. Red arrowheads indicate reduced expression of markers (b, $\mathbf{d}, \mathbf{f}, \mathbf{h})$. Shh and Ptcl expression in sagittal sections of the control and

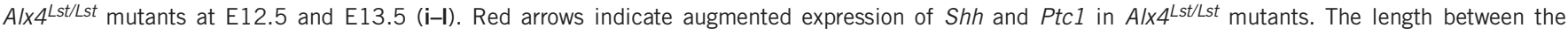
umbilical cord and the dorsal GT was measured at E18.5 ( $\mathrm{m}$; inset). The significant difference in the length is indicated by an asterisk. Results are presented as means $\pm \mathrm{SD}$. ${ }^{*} P<0.001$. gt: genital tubercle, hl: hindlimb, t: tail, uc: umbilical cord.

labeling experiment with $A l x 4^{L s t / L s t}$ mutants, we found that labeled cells in mutants migrated a shorter distance than those from control embryos (Figures $3 \mathrm{~g}$ and $\mathrm{h}$ ). These results suggest that cells in the proximal umbilical mesenchyme can migrate toward the GT mesenchyme and that this process is hampered by the Alx4 mutation.

\section{Genetic analysis of Alx4 and Hh signaling genes during GT formation}

The current results suggest that Alx4 regulates urogenital organ development. We next analyzed candidate genes that may interact with Alx4 in the developing urogenital system. Previous reports suggested a genetic interaction between Alx4 and Hh signaling during early limb development. ${ }^{38,39}$ We thus analyzed the genetic interaction between Alx4 and Hh signaling in the developing urogenital system. Fgf8 expression is a marker for the distal GT. ${ }^{15,44}$ Conventional and conditional mutants for Shh gene are known to display GT hypoplasia and reduced expression of $F g f 8$, suggesting a possibility of distal GT hypoplasia. ${ }^{15,17}$ Hence, we analyzed the expression of Fgf8 in the GT of Shh; Alx $4^{L s t}$ combinatorial mutants. In wild-type embryos, Fgf8 expression was detected in the distal GT (Supplementary Figures S1a and $\mathrm{a}^{\prime}$ ) and its expression was slightly augmented in the ventral GT of Alx $4^{\text {Lst/Lst }}$ mutants (Supplementary Figures S1b and $\mathrm{b}^{\prime}$ ). In contrast, $\mathrm{Shh}^{-/-}$mutants with GT agenesis did not display such expression (Supplementary Figures S1c and $c^{\prime}$ ). We next examined Fgf8 expression in double mutants of $A l x 4$ and Shh. Fgf8 expression was restored with slight protrusion of the GT in the mutants (Supplementary Figures S1d and $\mathrm{d}^{\prime}$; red arrows). These genetic analyses suggest that Alx4 can negatively regulate Hh signaling during GT formation.

\section{Defective GT formation by aberrant Hh signaling}

We then assessed the genetic interaction between Alx4 and Gli3, which mainly works as a negative regulator of Hh signaling, during GT formation. The introduction of Alx $4^{L s t}$ mutation into a Gli3 ${ }^{\mathrm{Xt} / \mathrm{Xt}}$ mutant background led to severe hypoplasia of the dorsal GT (Figures $4 \mathrm{a}-\mathrm{c}^{\prime}$; black arrows). The Gli3 ${ }^{\mathrm{X} / \mathrm{Xt}}$ single mutants displayed slight hypoplasia of dorsal GT (data not shown). An additional introduction of $A l x 4^{L s t}$ mutation to the Gli3 ${ }^{X t / X t}$ mutant background $\left(A l x 4^{L s t /+}\right.$; Gli3 ${ }^{X t / X t}$ mutants) resulted in hypoplasia of the dorsal GT (Figure 4b; black arrow). Furthermore, double homozygous mutants 


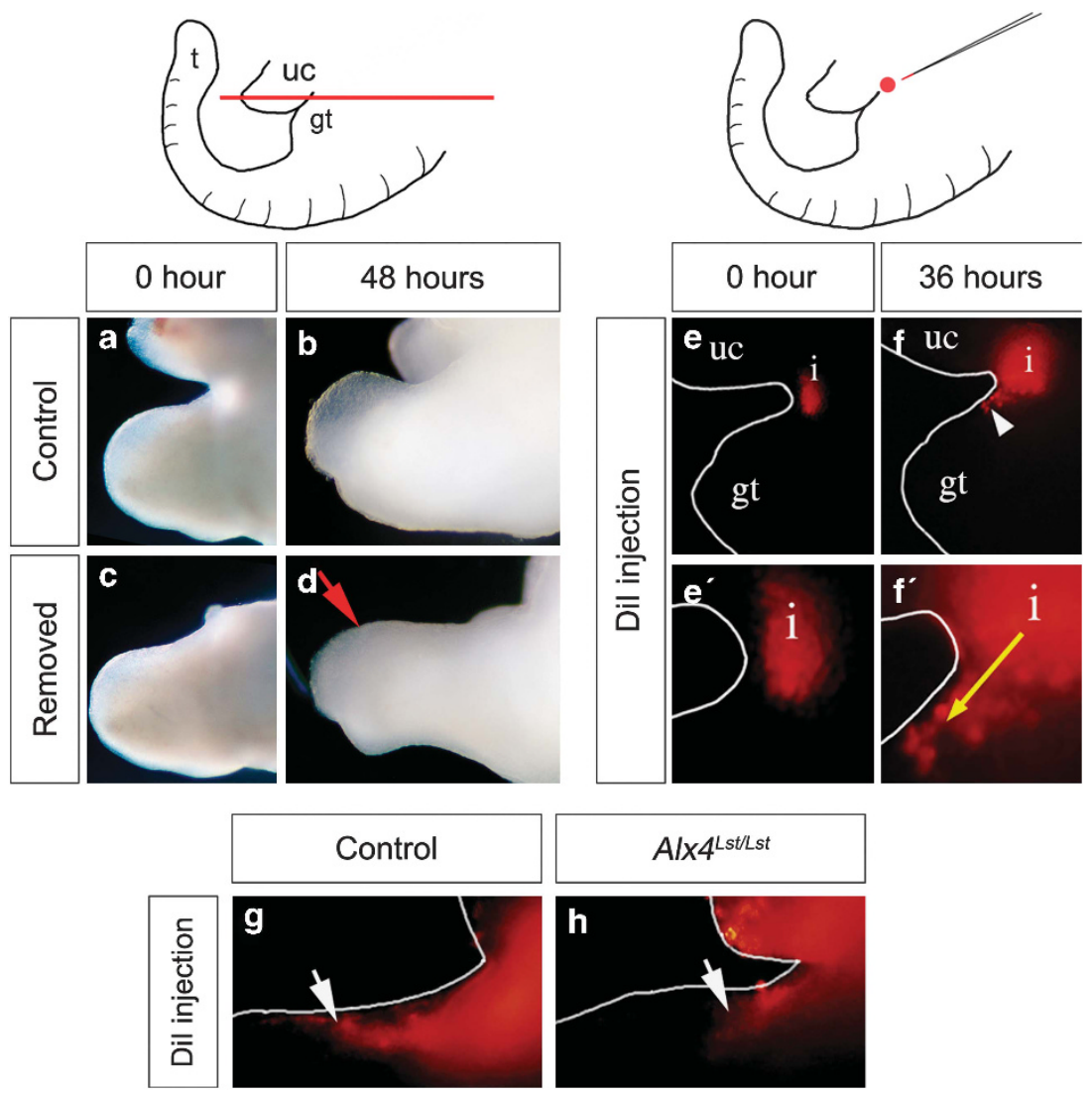

Figure 3 Tissue extirpation and cell migration of proximal umbilical mesenchyme. Surgical removal of the proximal part of umbilical cord at E12.5 ( $0 \mathrm{~h})$ (a, c) resulted in hypoplasia of the dorsal GT (b, d; red arrow). Dil was injected into the embryonic tissue slices at E12.0 (Oh) (e, $\left.\mathbf{e}^{\prime}\right)$ and the slices were cultured for $36 \mathrm{~h}\left(\mathbf{f}, \mathbf{f}^{\prime}\right)$. $\mathbf{e}^{\prime}$ and $\mathbf{f}^{\prime}$ are magnified views of $\mathbf{e}$ and $\mathbf{f}$. Yellow arrow indicates the direction of migration of Dil-labeled cells. Dil-injected tissues of Alx $4^{L s t / L s t}$ mutants displayed reduced migration (g, $\mathbf{h}$; white arrows). gt: genital tubercle, i: Dil, t: tail, uc: umbilical cord.

$\left(A l x 4^{L s t / L s t} ; G l i 3^{X t / X t}\right)$ displayed more severe hypoplasia of both dorsal and ventral GT structures (Figure 4c; black arrows). These mutants also displayed hypoplastic body wall and a shortened distance between the umbilical cord and GT at E15.5 (Figures $4 \mathrm{a}^{\prime}-\mathrm{c}^{\prime}$; blue arrows). The expression of several marker genes such as Pitxl and Mab21l2 was reduced in the upper cloacal mesenchyme in $A l x 4^{L s t / L s t}$; Gli3 ${ }^{X t / X t}$ mutants (Supplementary Figures S2a-d; red arrowheads).

Alx $4^{\text {Lst/Lst }}$; Gli3 ${ }^{X t /+}$ mutants also displayed dorsal GT hypoplasia with ventral body wall abnormalities (Figures $4 \mathrm{~d}-\mathrm{f}$; red arrows and blue arrowhead). Notably, decreasing Shh gene dosage partially rescued the morphology of the GT and body wall in Alx $4^{\text {Lst } / L s t}$; $\mathrm{Gli3}^{\mathrm{Xt} /+} ; \mathrm{Shh}^{+/-}$mutants (Figures $4 \mathrm{~g}-\mathrm{i}$ ). These data are all consistent with increased Shh signaling contributing to the complex phenotypes of Alx4 mutants.

We performed complementary experiments by using R26-SmoM2 allele, which possesses a constitutively active form of Smoothened (SmoM2) and a floxed stop cassette under the ubiquitous Rosa26 promoter. ${ }^{32,34}$ By crossing R26-SmoM2 female mice and CAGGS-CreER ${ }^{T M}$ transgenic male mice, an augmentation of Hh signaling is achieved in embryos after induction with tamoxifen. ${ }^{28}$ Tamoxifen treatment at E10.5 resulted in hypoplastic prepuce and uncovered the glans penis of the mutant GT at E17.5 (Figure 4k; red arrowhead). These results further support our hypothesis that excess $\mathrm{Hh}$ signaling could contribute to dorsal GT hypoplasia.

\section{DISCUSSION}

Although the causative genes and mutations for inherited urogenital diseases have been identified, ${ }^{45}$ the functions of these genes in complex syndromes remain elusive. In particular, cellular mechanisms for tissue coordination during development of the urogenital organs are largely unknown, even though discoordination likely contributes to malformation of these structures. We previously reported several mutants with body wall defects associated with abdominal muscle and pelvic girdle abnormalities. ${ }^{28}$ Here we show that Alx4 mutants have reduced expression of Ap2 $\alpha$, Mab21l2 and Pitxl genes, all of which are essential for body wall and/or pelvic girdle formation. ${ }^{33,35,46}$ In the case of $A l x 4^{L s t}$; Gli3 ${ }^{X t}$ combinatorial mutants, the severities of GT phenotypes correlate to those of the body wall and pelvic girdle. From these observations, we suggest that the GT, the ventral body wall and the pelvic girdle develop coordinately. In addition, we also showed that cell migration is crucial for proper GT formation and is a likely cellular mechanism for coordinating the development of neighboring urogenital tissues. The current study suggests part of the pathogenic mechanisms for the broad-spectrum symptoms of some clinical patients.

Bladder exstrophy (OMIM: \%600057) is a complex birth defect often associated with abnormalities of the external genitalia, rectus muscles and separation of the pubic bones. ${ }^{4}$ In addition, the umbilical cord is aberrantly located immediately above the bladder. ${ }^{1}$ Such features resemble the phenotypes of $A l x 4^{\text {Lst/Lst }}$ mouse mutants. 

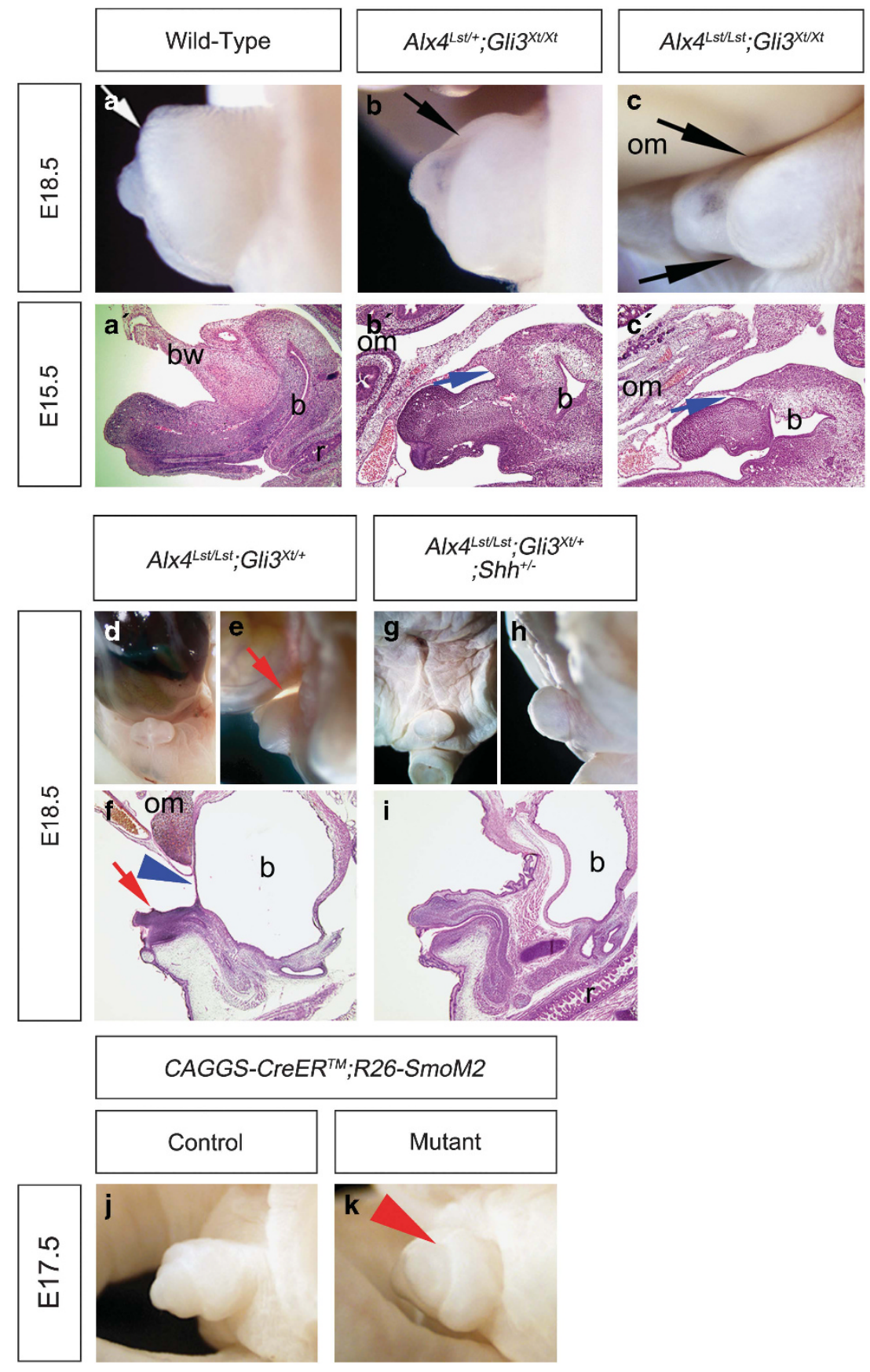

Figure 4 Genetic interaction between $A / x 4$ and the Hedgehog signaling pathway during lower body formation. Lateral view and sagittal sections of wild-type,



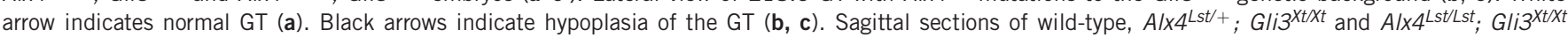
embryos at E15.5 $\left(\mathbf{a}^{\prime}-\mathbf{c}^{\prime}\right)$. Blue arrows in panels $\mathbf{b}^{\prime}$ and $\mathbf{d}^{\prime}$ indicate hypoplasia of the ventral body wall. Frontal $(\mathbf{d}, \mathbf{g})$ and lateral $(\mathbf{e}, \mathbf{h})$ view of the GT in

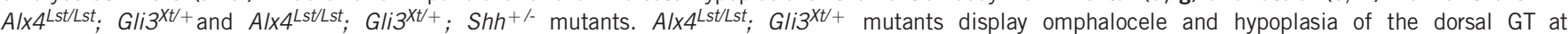


Gli3 ${ }^{X t+}$; Shh $h^{+/}$mutants $(\mathbf{f}, \mathbf{i})$. Red arrows in panels e and $\mathbf{f}$ indicate hypoplasia of the dorsal GT. Blue arrowhead indicates hypoplastic ventral body wall in Alx4 ${ }^{L s t / L s t}$; Gli3 ${ }^{X t+}+$ mutants. Lateral view of the GT of Hh gain-of-function mutants at E17.5 (j, k). Red arrowhead indicates hypoplasia of prepuce (k). b: bladder, bw: body wall, om: omphalocele, r: rectum.

Additional phenotypes including craniofacial abnormalities have been reported in both human patients and Alx $4^{\text {Lst/Lst }}$ mouse mutants. However, human patients and mouse mutants often do not display identical phenotypes. Even in the case of human patients, there is significant variability in phenotypes resulting from different $A L X 4$ mutations, such as nonsyndromic craniosynostosis by V7F and $\mathrm{K} 211 \mathrm{E}$ mutations and syndromic craniofacial abnormalities including hypertelorism and cryptorchidism (undescended testis) with homozygous R265X mutation. ${ }^{25,47}$ A case report with deletion of chromosome $11 \mathrm{p} 12$ extending to bands p11.2 and p13 (ALX4 is 

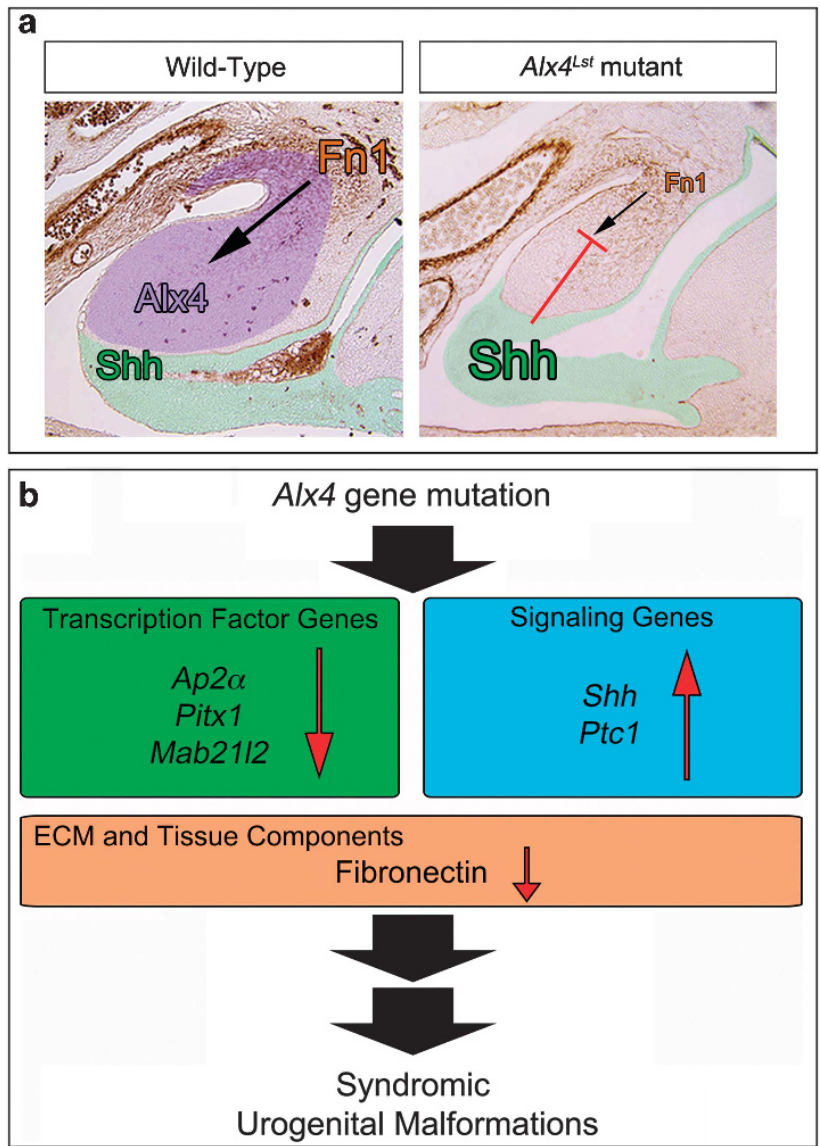

Figure 5 The summary schemes (a, b). (a) Brown signal indicates Fibronectin ( $F n 1)$ expression. The $A / x 4$-positive region is marked in purple. Shh expression is marked in green. Cells in the proximal umbilical mesenchyme migrate toward the GT region in wild-type embryos (black arrow). The introduction of mutation in $\mathrm{A} / \mathrm{x} 4$ gene affects $\mathrm{Hh}$ signaling with reduced Fibronectin ( $\mathrm{Fn} 1$ ) expression, leading to defective cell migration (right panel). (b) The Alx4 mutation resulted in the reduced expression or expression area of several transcription factor genes such as $A p 2 \alpha$, Pitx 1 and Mab21/2. On the other hand, the expression of Shh and Ptc1 was augmented. In one of the extracellular matrix and tissue components, fibronectin was also downregulated. As a result, it is suggested that $A / x 4$ mutants displayed syndromic urogenital malformation.

located on 11p11.2) had micropenis phenotype in addition to mild facial dysmorphism. ${ }^{48}$ Therefore, additional, as yet unidentified, mutations such as those seen in $A l x 4^{L s t}$ (Lst): a 16-bp deletion within the paired-type homeodomain, which causes a frame shift potentially producing a truncated protein at amino acid position 332) may cause urogenital syndromic malformations in humans. Additional genetic events, such as copy number variation or epigenetic modifications of ALX4, may also cause phenotypes in humans. Recently, genome-wide copy number variation studies have been performed for anorectal malformations and have identified some affected genes; ${ }^{49}$ however, the importance of these findings is not yet clear. On the other hand, ALX4 is frequently methylated in adenocarcinomas of the gastrointestinal tract. ${ }^{50}$ Hence, such human studies may become more essential for human ALX4 analyses.

The current analyses speculate that the loss of proximal umbilical cells and/or the disturbance of their migration toward the dorsal GT may contribute to syndromic malformations of structures derived from these tissues by utilizing Alx4 and Hh signaling (Figure 5). Such pathogenic mechanisms are likely applicable to other genes that affect cell migration and cell number in the proximal umbilical mesenchyme. In response to the growing number of infertility patients, assisted reproductive technology such as in vitro fertilization is becoming increasingly common. The exstrophy-epispadias complex is known to occur more frequently in children conceived by in vitro fertilization. ${ }^{51}$ We believe the current study will contribute to understanding the basis for such defects. Further clinical analyses of patients together with model animal studies will reveal the pathogenic mechanisms for human syndromic malformations.

\section{CONFLICT OF INTEREST}

The authors declare no conflict of interest.

\section{ACKNOWLEDGEMENTS}

We would like to specially thank Drs Frits Meijlink, Trevor Williams, Andrew P McMahon, Chi-chung Hui, Hiroshi Sasaki, Sanne Kuijper, Toshihiko Shiroishi, Chin Chiang and Philippe Soriano for their invaluable support. We would also like to thank Drs Pierre Chambon, Brigid LM Hogan, YiPing Chen, Jun Motoyama, Chisa Shukunami and Rolf Zeller for their encouragement and suggestions. We would also like to express our appreciation to Sawako Fujikawa and Tomiko I Iba for their assistance. This work is supported by Grant-in-Aid for Young Scientists B (23790228 and 24790292) and for Scientific Research on Innovative Areas: Molecular mechanisms for establishment of sex differences (22132006) from the Ministry of Education, Culture, Sports, Science, and Technology, Japan. This work is also supported by the National Institutes of Health Grant R01ES016597.

1 Perovic SV: Atlas of Congenital Anomalies of the External Genitalia. Belgrade, Yugoslavia: Refot-Arka, 1999

2 Ebert A, Reutter H, Ludwig M, Rösch W: The exstrophy-epispadias complex. Orphanet J Rare Dis 2009; 4: 23.

3 Ludwig M, Ching B, Reutter H, Boyadjiev S: Bladder exstrophy-epispadias complex. Birth Defects Res A Clin Mol Teratol 2009; 85: 509-522.

4 Campbell MF, Walsh PC, Retik AB: Campbell's Urology, 8th edn. Philadelphia, PA Saunders, 2002.

5 Mann S, Blinman T, Douglas Wilson R: Prenatal and postnatal management of omphalocele. Prenat Diagn 2008; 28: 626-632.

6 Aspelund G, Langer J: Abdominal wall defects. Curr Paediatr 2006; 16: 192-198.

7 Li MM, Andersson HC: Clinical application of microarray-based molecular cytogenetics: an emerging new era of genomic medicine. J Pediatr 2009; 155: 311-317.

8 van Ommen GJ: The Human Genome Project and the future of diagnostics, treatment and prevention. J Inherit Metab Dis 2002; 25: 183-188.

9 Zouvelou V, Passa O, Segklia K et al: Generation and functional characterization of mice with a conditional BMP7 allele. Int J Dev Biol 2009; 53: 597-603.

10 Penington EC, Hutson JM: The cloacal plate: the missing link in anorectal and urogenital development. BJU Int 2002; 89: 726-732.

11 Baskin LS, Himes K, Colborn T: Hypospadias and endocrine disruption: is there a connection? Environ Health Perspect 2001; 109: 1175-1183.

12 Lin C, Yin Y, Veith GM, Fisher AV, Long F, Ma L: Temporal and spatial dissection of Shh signaling in genital tubercle development. Development 2009; 136: 3959-3967.

13 Miyagawa S, Satoh Y, Haraguchi R et al: Genetic interactions of the androgen and Wnt/ beta-catenin pathways for the masculinization of external genitalia. Mol Endocrinol 2009; 23: 871-880.

14 Haraguchi R, Motoyama J, Sasaki H et al: Molecular analysis of coordinated bladder and urogenital organ formation by Hedgehog signaling. Development 2007; 134: 525-533.

15 Miyagawa S, Moon A, Haraguchi $\mathrm{R}$ et al: Dosage-dependent hedgehog signals integrated with Wnt/beta-catenin signaling regulate external genitalia formation as an appendicular program. Development 2009; 136: 3969-3978.

16 Miyagawa S, Matsumaru D, Murashima A et al: The role of sonic hedgehog-Gli2 pathway in the masculinization of external genitalia. Endocrinology 2011; 152: 2894-2903.

17 Haraguchi R, Mo R, Hui C et al: Unique functions of Sonic hedgehog signaling during external genitalia development. Development 2001; 128: 4241-4250.

18 Seifert AW, Bouldin CM, Choi KS, Harfe BD, Cohn MJ: Multiphasic and tissue-specific roles of sonic hedgehog in cloacal septation and external genitalia development. Development 2009; 136: 3949-3957.

19 van der Zanden LF, van Rooij IA, Feitz WF et al: Common variants in DGKK are strongly associated with risk of hypospadias. Nat Genet 2011; 43: 48-50. 
20 Qu S, Niswender KD, Ji Q et al: Polydactyly and ectopic ZPA formation in Alx-4 mutant mice. Development 1997; 124: 3999-4008.

21 Beverdam A, Brouwer A, Reijnen M, Korving J, Meijlink F: Severe nasal clefting and abnormal embryonic apoptosis in Alx3/Alx4 double mutant mice. Development 2001; 128: 3975-3986.

22 Wu YQ, Badano JL, McCaskill C, Vogel H, Potocki L, Shaffer LG: Haploinsufficiency of ALX4 as a potential cause of parietal foramina in the $11 \mathrm{p} 11.2$ contiguous genedeletion syndrome. Am J Hum Genet 2000; 67: 1327-1332.

23 Wuyts W, Cleiren E, Homfray T, Rasore-Quartino A, Vanhoenacker F, Van Hul W: The ALX4 homeobox gene is mutated in patients with ossification defects of the skull (foramina parietalia permagna, OMIM 168500). J Med Genet 2000; 37: 916-920.

24 Kayserili H, Altunoglu U, Ozgur H, Basaran S, Uyguner ZO: Mild nasal malformations and parietal foramina caused by homozygous ALX4 mutations. Am J Med Genet $A$ 2012; 158A: 236-244.

25 Kayserili H, Uz E, Niessen C et al: ALX4 dysfunction disrupts craniofacial and epidermal development. Hum Mol Genet 2009; 18: 4357-4366.

26 Qu S, Tucker SC, Ehrlich JS et al: Mutations in mouse Aristaless-like4 cause Strong's luxoid polydactyly. Development 1998; 125: 2711-2721.

27 Takahashi M, Tamura K, Buscher D et al: The role of Alx-4 in the establishment of anteroposterior polarity during vertebrate limb development. Development 1998; 125 : 4417-4425.

28 Matsumaru D, Haraguchi R, Miyagawa S, Motoyama J, Nakagata N, Sham MH: Genetic analysis of hedgehog signaling in ventral body wall development and the onset of omphalocele formation. PLOS ONE 2011; 6: 1307-1321.

29 Chiang C, Litingtung Y, Lee $\mathrm{E}$ et al: Cyclopia and defective axial patterning in mice lacking sonic hedgehog gene function. Nature 1996; 383: 407-413.

30 Hui CC, Joyner AL: A mouse model of greig cephalopolysyndactyly syndrome: the extratoesJ mutation contains an intragenic deletion of the Gli3 gene. Nat Genet 1993; 3: 241-246.

31 Hayashi S, McMahon AP: Efficient recombination in diverse tissues by a tamoxifeninducible form of Cre: a tool for temporally regulated gene activation/inactivation in the mouse. Dev Biol 2002; 244: 305-318.

32 Mao J, Ligon KL, Rakhlin EY et al: A novel somatic mouse model to survey tumorigenic potential applied to the Hedgehog pathway. Cancer Res 2006; 66: 10171-10178.

33 Yamada R, Mizutani-Koseki Y, Koseki H, Takahashi N: Requirement for Mab2112 during development of murine retina and ventral body wall. Dev Biol 2004; 274: 295-307.

34 Jeong J, Mao J, Tenzen T, Kottmann AH, McMahon AP: Hedgehog signaling in the neural crest cells regulates the patterning and growth of facial primordia. Genes Dev 2004; 18: 937-951.

35 Brewer S, Williams T: Loss of AP-2alpha impacts multiple aspects of ventral body wall development and closure. Dev Biol 2004; 267: 399-417.

36 Schorle H, Meier P, Buchert M, Jaenisch R, Mitchell P: Transcription factor AP-2 essential for cranial closure and craniofacial development. Nature 1996; 381: 235-238.
37 Zhang J, Hagopian-Donaldson S, Serbedzija G et al: Neural tube, skeletal and body wall defects in mice lacking transcription factor AP-2. Nature 1996; 381: 238-241.

38 Kuijper S, Feitsma H, Sheth R, Korving J, Reijnen M, Meijlink F: Function and regulation of Alx4 in limb development: complex genetic interactions with Gli3 and Shh. Dev Biol 2005; 285: 533-544.

39 Panman L, Drenth T, Tewelscher P, Zuniga A, Zeller R: Genetic interaction of Gli3 and Alx4 during limb development. Int J Dev Biol 2005; 49: 443-448.

40 Pearse RV, Vogan KJ, Tabin CJ: Ptc1 and Ptc2 transcripts provide distinct readouts of Hedgehog signaling activity during chick embryogenesis. Dev Biol 2001; 239: 15-29.

41 Ingham PW: Transducing hedgehog: the story so far. EMBO J 1998; 17: 3505-3511.

42 Gilboa Y, Katorza E, Kedem A, Spira M, Achiron R: Measurement of the fetal umbilical cord insertion-to-genital tubercle length in early gestation: in utero sonographic study. J Ultrasound Med 2011; 30: 237-241.

43 Pankov R, Yamada KM: Fibronectin at a glance. J Cell Sci 2002; 115: 3861-3863.

44 Haraguchi R, Suzuki K, Murakami R et al: Molecular analysis of external genitalia formation: the role of fibroblast growth factor (Fgf) genes during genital tubercle formation. Development 2000; 127: 2471-2479.

45 Fisher SE, Black GC, Lloyd SE et al: Isolation and partial characterization of a chloride channel gene which is expressed in kidney and is a candidate for Dent's disease (an X-linked hereditary nephrolithiasis). Hum Mol Genet 1994; 3: 2053-2059.

46 Szeto DP, Rodriguez-Esteban C, Ryan AK et al: Role of the Bicoid-related homeodomain factor Pitx1 in specifying hindlimb morphogenesis and pituitary development. Genes Dev 1999; 13: 484-494.

47 Yagnik G, Ghuman A, Kim S et al: ALX4 gain-of-function mutations in nonsyndromic craniosynostosis. Hum Mutat 2012; 33: 1626-1629.

48 Bartsch O, Wuyts W, Van Hul W et al: Delineation of a contiguous gene syndrome with multiple exostoses, enlarged parietal foramina, craniofacial dysostosis, and mental retardation, caused by deletions in the short arm of chromosome 11. Am J Hum Genet 1996; 58: 734-742.

49 Wong $\mathrm{EH}$, Cui L, Ng CL et al: Genome-wide copy number variation study in anorectal malformations. Hum Mol Genet 2013; 22: 621-631.

50 Ebert MP, Model F, Mooney S et al: Aristaless-like homeobox-4 gene methylation is a potential marker for colorectal adenocarcinomas. Gastroenterology 2006; 131: $1418-1430$.

51 Wood HM, Trock BJ, Gearhart JP: In vitro fertilization and the cloacal-bladder exstrophy-epispadias complex: is there an association? J Urol 2003; 169: $1512-1515$.

\footnotetext{
(c) (i) $(-)$ This work is licensed under a Creative Commons Attribution-NonCommercial-NoDerivs 3.0 Unported License. To view a copy of this license, visit http://creativecommons. org/licenses/by-nc-nd/3.0/
}

Supplementary Information accompanies this paper on European Journal of Human Genetics website (http://www.nature.com/ejhg) 